\title{
Observation of anti-damping spin-orbit torques generated by in-plane and out-of-plane spin polarizations in MnPd3
}

Mahendra DC ( $\sim$ mdc2019@stanford.edu )

Stanford University https://orcid.org/0000-0003-1249-336X

Ding-Fu Shao

University of Nebraska

Vincent Hou

Taiwan Semiconductor Manufacturing Company (Taiwan)

Patrick Quarterman

NIST Center for Neutron Research https://orcid.org/0000-0001-6272-6243

Ali Habiboglu

University of Arizona

Brooks Venuti

Colorado School of Mines

Masashi Miura

Seikei University

Brian Kirby

NIST https://orcid.org/0000-0003-2472-3791

Arturas Vailionis

Stanford Nano Shared Facilities, Stanford University https://orcid.org/0000-0001-5878-1864

Chong Bi

Institute of Microelectronics Chinese Academy of Science

\section{Xiang Li}

Stanford University

Fen Xue

Stanford University

Yen-Lin Huang

TSMC

\section{Yong Deng}

Stanford University

Shy-Jay Lin

Taiwan Semiconductor Manufacturing Company (Taiwan)

\section{Wilman Tsai}


Stanford University

\section{Serena Eley}

Colorado School of Mines https://orcid.org/0000-0002-2928-5316

\section{Weigang Wang}

University of Arizona

Julie Borchers

NCNR/NIST https://orcid.org/0000-0002-3348-8212

\section{Evgeny Tsymbal}

University of Nebraska-Lincoln https://orcid.org/0000-0002-6728-5480

\section{Shan Wang}

Stanford University

\section{Article}

Keywords: Magnetic Memory, Logic Devices, External Magnetic Field-free Switching, Pracitical Spin Channel

Posted Date: January 8th, 2021

DOI: https://doi.org/10.21203/rs.3.rs-132828/v1

License: (c) (i) This work is licensed under a Creative Commons Attribution 4.0 International License. Read Full License 


\title{
Observation of anti-damping spin-orbit torques generated by in-plane and out-of-plane spin polarizations in $\mathrm{MnPd}_{3}$
}

\author{
Mahendra DC ${ }^{*}$, Ding-Fu Shao ${ }^{2}$, Vincent D.-H. Hou $^{3}$, P. Quarterman ${ }^{4}$, Ali Habiboglu ${ }^{5}$, Brooks \\ Venuti $^{6}$, Masashi Miura ${ }^{1,7}$, Brian Kirby ${ }^{4}$, Arturas Vailionis ${ }^{8,9}$, Chong $\mathrm{Bi}^{10}$, Xiang $\mathrm{Li}^{1,10}$, Fen \\ Xue $^{1}$, Yen-Lin Huang ${ }^{3}$, Yong Deng ${ }^{1}$, Shy-Jay Lin ${ }^{3}$, Wilman Tsai ${ }^{1}$, Serena Eley ${ }^{6}$, Weigang \\ Wang $^{5}$, Julie A. Borchers ${ }^{4}$, Evgeny Y. Tsymbal ${ }^{2}$, and Shan X. Wang ${ }^{1,10 *}$ \\ ${ }^{1}$ Department of Materials Science and Engineering, Stanford University, Stanford, California 94305, USA \\ ${ }^{2}$ Department of Physics and Astronomy \& Nebraska Center for Materials and Nanoscience, University of Nebraska, \\ Lincoln, NE 68588-0299 \\ ${ }^{3}$ Taiwan Semiconductor Manufacturing Company, Hsinchu, Taiwan \\ ${ }^{4}$ NIST Center for Neutron Research, National Institute of Standards and Technology, 100 Bureau Dr., Gaithersburg, \\ Maryland 20899, USA \\ ${ }^{5}$ Department of Physics, University of Arizona, Tucson, Arizona 85721, USA \\ ${ }^{6}$ Department of Physics, Colorado School of Mines, Golden, Colorado 80401 \\ ${ }^{7}$ Graduate School of Science and Technology, Seikei University, 3-3-1 Kichijoji-kitamachi, Musashinoshi, Tokyo, \\ 180-8633, Japan \\ ${ }^{8}$ Stanford Nano Shared Facilities, Stanford University, Stanford, CA 94305, USA \\ ${ }^{9}$ Department of Physics, Kaunas University of Technology, LT-51368 Kaunas, Lithuania \\ ${ }^{10}$ Department of Electrical Engineering, Stanford University, Stanford, California 94305, USA
}

High spin-orbit torques (SOTs) generated by topological materials and heavy metals interfaced with a ferromagnetic layer show promise for next generation magnetic memory and logic devices. SOTs generated from the in-plane spin polarization along $y$-axis originated by the spin Hall and Edelstein effects can switch magnetization collinear with the spin polarization in the absence of external magnetic fields. However, an external magnetic field is required to switch the magnetization along $x$ and $z$-axes via SOT generated by $y$-spin polarization. Here, we present that the above limitation can be circumvented by unconventional SOT in magnetron-sputtered thin film $\mathrm{MnPd}_{3}$. In 
31 addition to the conventional in-plane anti-damping-like torque due to the $y$-spin 32 polarization, out-of-plane and in-plane anti-damping-like torques originating from $z$-spin 33 and $x$-spin polarizations, respectively have been observed at room temperature. The spin 34 torque efficiency $\left(\theta_{y}\right)$ corresponding to the $y$-spin polarization from $\mathrm{MnPd}_{3}$ thin films 35 grown on thermally oxidized silicon substrate and post annealed at $400{ }^{\circ} \mathrm{C}$ is $0.34-0.44$ 36 while the spin conductivity $\left(\sigma_{z x}^{y}\right)$ is $\sim 5.70-7.30 \times 10^{5} \hbar / 2 e \Omega^{-1} \mathbf{m}^{-1}$. Remarkably, we have 37 demonstrated complete external magnetic field-free switching of perpendicular Co layer 38 via unconventional out-of-plane anti-damping-like torque from $z$-spin polarization. Based 39 on the density functional theory calculations, we determine that the observed $x$ - and $z$ - spin 40 polarizations with the in-plane charge current are due to the low symmetry of the (114) 41 oriented $\mathrm{MnPd}_{3}$ thin films. Taken together, the new material reported here provides a path 42 to realize a practical spin channel in ultrafast magnetic memory and logic devices. 
Efficient control of magnetization at ultra-high speed has been of prime interest to the

47 spintronics community ${ }^{1}$. Spin-orbit torque (SOT) has provided efficient and ultrafast control of

48 magnetization in magnetoresistive random access memory (MRAM) and logic devices ${ }^{2,3}$. SOT

49 has been observed in magnetic semiconductor ${ }^{4}$ and heavy metals ${ }^{5-7}$, topological insulators ${ }^{8-12}$, 50 antiferromagnets ${ }^{13-17}$, and semimetals ${ }^{18-20}$ interfaced with a ferromagnetic layers. The charge

51 current injected into the non-magnetic layer (spin channel) along the $x$-direction generates a spin

52 current along $z$-direction with spin polarization $(\hat{\sigma})$ pointing along $y$-direction $\left(\hat{\sigma}_{y}\right)$ in heavy

53 metals due to the bulk spin Hall effect. In the case of topological insulators and Weyl semimetals

54 non-equilibrium spin-density is accumulated at the interface due to the time reversal symmetry

55 protected spin momentum locking ${ }^{21}$. SOTs exerted on the ferromagnet with in-plane magnetic

56 anisotropy (IMA) are in-plane anti-damping-like $\left(\boldsymbol{\tau}_{\boldsymbol{A D L}, \boldsymbol{y}} \propto \widehat{m} \times\left(\hat{\sigma}_{y} \times \widehat{m}\right)\right)$ and out-of-plane

57 field-like $\left(\boldsymbol{\tau}_{\boldsymbol{F} \boldsymbol{L}} \propto \hat{\sigma}_{y} \times \widehat{m}\right)$, where $\widehat{m}$ is magnetization unit vector. Spin current with $\hat{\sigma}$ along $z-$

58 direction $\left(\hat{\sigma}_{z}\right)$ has been observed in transition-metal dichalcogenides ${ }^{18,20,22}$ and, ferromagnets

59 interfaced with light metals ${ }^{23}$ with the charge current flow along $x$-direction. SOT due to $\hat{\sigma}_{z}$

60 exerts torque along the out-of-plane direction $\left(\boldsymbol{\tau}_{\boldsymbol{A D L}, \boldsymbol{z}} \propto \widehat{m} \times\left(\hat{\sigma}_{z} \times \widehat{m}\right)\right)$, which will enable

61 external field free and low power switching of the out-of-plane magnetization ${ }^{24}$. Recently, $\hat{\sigma}$

62 along the $x$-direction $\left(\hat{\sigma}_{x}\right)$ has been reported in the uncompensated antiferromagnet $\mathrm{Mn}_{3} \mathrm{GaN}$ in

63 addition to $\hat{\sigma}_{y}$ and $\hat{\sigma}_{z}$ due to the low magnetic symmetry ${ }^{17}$. SOT due to $\hat{\sigma}_{x}$ exerts torque along in-

64 plane direction $\left(\boldsymbol{\tau}_{\boldsymbol{A D L}, \boldsymbol{x}} \propto \widehat{m} \times\left(\hat{\sigma}_{x} \times \widehat{m}\right)\right)$, which will deterministically switch the magnetization

65 along the $x$-direction in the absence of external magnetic field. The figure of merit of charge to

66 spin conversion is known as the spin torque efficiency $\theta_{k} \propto \frac{\sigma_{i j}^{k}}{\sigma_{x x}}$, where $\sigma_{i j}^{k}(i, j$, and $k$ refers to

67 the spin current flow, charge current flow, and spin polarization directions, respectively) and $\sigma_{x x}$ 
are spin and charge conductivities, respectively. $\theta_{k}$ needs to be high for efficient control of the magnetization. Furthermore, to avoid current shunting through a conducting ferromagnetic layer high $\sigma_{i j}^{k}$ is also required ${ }^{25}$. Another important requirement for the integration of a spin channel into semiconductor IC technology is the tolerance of SOT materials to thermal annealing at 400

$72{ }^{\circ} \mathrm{C}$. However, there has not been a practical spin channel which can handle post annealing at that temperature, and also possesses high $\boldsymbol{\tau}_{\boldsymbol{A D L}, \boldsymbol{y}}$ along with $\boldsymbol{\tau}_{\boldsymbol{A D L}, \boldsymbol{x}}$ and $\boldsymbol{\tau}_{\boldsymbol{A D L}, \boldsymbol{z}}$, enabling deterministic

74 switching of in-plane magnetization along $y$, in-plane magnetization along $x$, and out-of-plane 75 magnetization, respectively, without the need of applying an external magnetic field.

To achieve high density magnetic memory and logic devices, perpendicular magnetic 77 anisotropy (PMA) is desired ${ }^{26}$. PMA switching via SOT from heavy metals ${ }^{5-7}$ and topological 78 insulators ${ }^{11,12,27,28}$ has been reported at the room temperature in the presence of an external 79 magnetic field. Partial PMA switching has been observed on $\mathrm{PtMn} /[\mathrm{Co} / \mathrm{Ni}]_{\mathrm{x}}{ }^{13}, \mathrm{IrMn} / \mathrm{CoFeB}{ }^{14}$, and $\mathrm{PtMn} / \mathrm{CoFeB} / \mathrm{Gd} / \mathrm{CoFeB}^{16}$ stack structures, in the absence of external magnetic field, but

81 with the help of exchange bias. Stray fields from an in-plane magnetic layer present above or 82 below the spin channel can facilitate external magnetic field-free PMA switching, but current 83 shunting and magnetic interference between different magnetic layers pose severe design 84 constraints in this approach ${ }^{29,30}$. Combination of SOT and spin transfer torque (STT) can also 85 switch PMA in the absence of external magnetic field, but STT could lead to reduced endurance 86 of magnetic tunnel barrier and slower magnetization switching ${ }^{31}$. Fast switching of the 87 magnetization with lower critical current densities $\left(J_{s w}\right)$ can be achieved when the charge 88 current flow and magnetization are collinear, however, this geometry still requires an external 89 magnetic field to achieve magnetization switching ${ }^{7}$. Here, we present SOT from sputtered $\operatorname{MnPd}_{3}$ 90 thin films post annealed at $400{ }^{\circ} \mathrm{C}$ that can generate a spin current with $\hat{\sigma}$ along all three axes 
91 due to the charge current flow along $x$-direction, which represents a major advance over the

92 literature and overcomes significant limitations of the existing SOT materials.
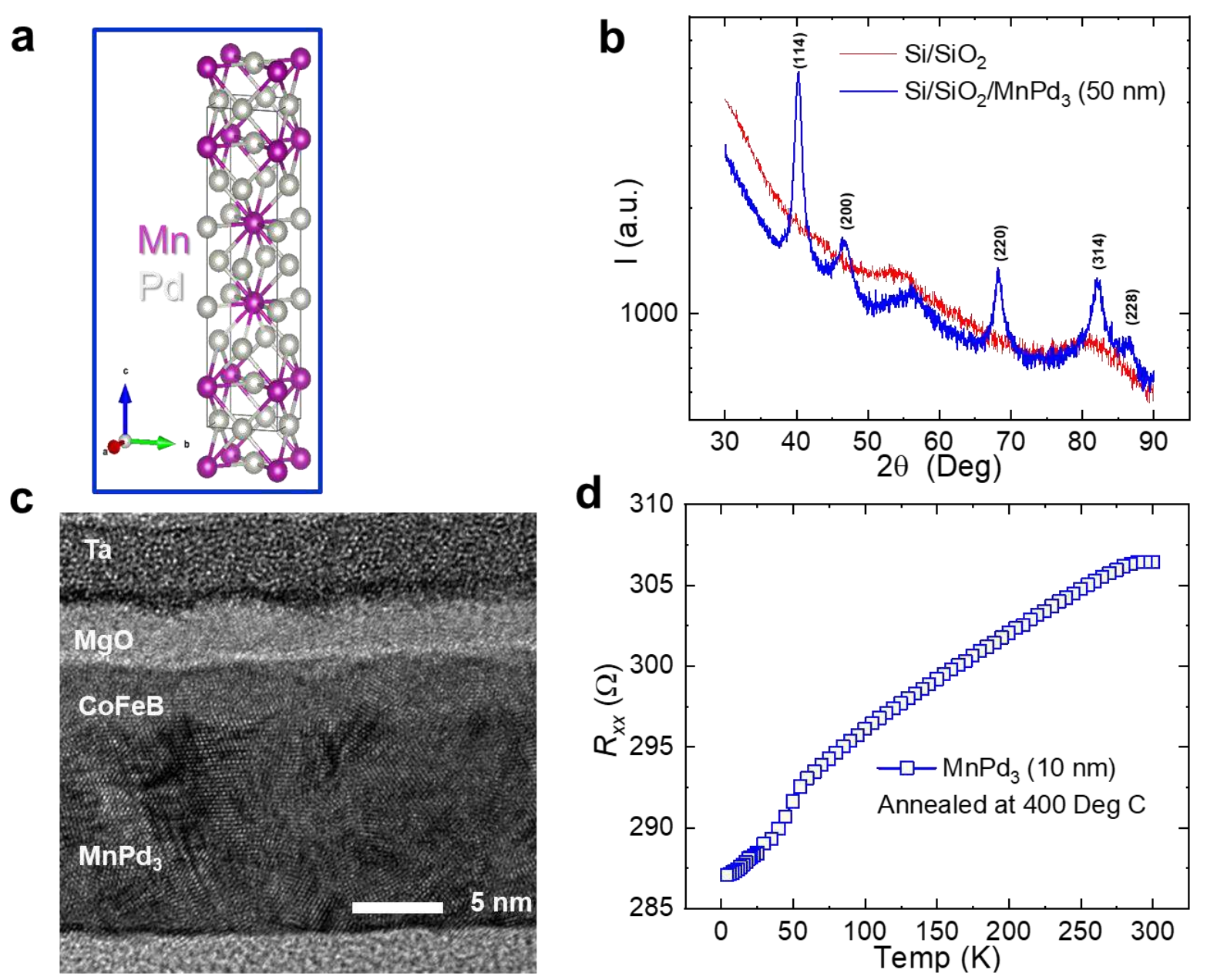

95

96 Fig. 1. Characterization of $\mathrm{MnPd}_{3}$ thin film: a, Schematic diagram of $\mathrm{D} 023 \mathrm{MnPd}_{3}$ unit cell.

$97 \mathbf{b}, \mathrm{XRD}$ of $\mathrm{Si} / \mathrm{SiO}_{2} / \mathrm{MnPd}_{3}(50 \mathrm{~nm})$ film, post-annealed at $400{ }^{\circ} \mathrm{C}$ for $30 \mathrm{~min}$. c, Cross-section

$98 \mathrm{TEM}$ image of $\mathrm{Si} / \mathrm{SiO}_{2} / \mathrm{MnPd}_{3}(10 \mathrm{~nm}) / \mathrm{CoFeB}(5 \mathrm{~nm}) / \mathrm{MgO}(2 \mathrm{~nm}) / \mathrm{Ta}(2 \mathrm{~nm})$ sample. d, Four

99 terminal resistance as a function of temperature of $\mathrm{Si} / \mathrm{SiO}_{2} / \mathrm{MnPd}_{3}(10 \mathrm{~nm})$ sample. 
The $\mathrm{MnPd}_{3}$ thin films were magnetron sputtered at room temperature on $300 \mathrm{~nm}$ thick

101

102

103

104

105

106

107

108

109

110

111

112

113

114

115

116

117

118

119

120

121 $122 \times 10^{22} / \mathrm{cm}^{3}$. At room temperature the values of anisotropic magnetoresistance (AMR) and planar

thermally oxidized silicon substrates. The thin films with the stack structure $\mathrm{Si} / \mathrm{SiO}{ }_{2} / \mathrm{MnPd}_{3}(t$ $\mathrm{nm}) / \mathrm{CoFeB} \quad(5 \mathrm{~nm}) / \mathrm{MgO}(2 \mathrm{~nm}) / \mathrm{Ta} \quad(2 \mathrm{~nm})$ with IMA were prepared for the SOT characterization with $t=4,6,8,10,12,16,20$, and $24 \mathrm{~nm}$, respectively. Unless otherwise stated, these films will be labeled MP4 - MP24, in which the number denotes the $\mathrm{MnPd}_{3}$ thickness. All samples were post annealed at $400{ }^{\circ} \mathrm{C}$ for 30 minutes. Using Rutherford backscattering, the atomic composition of $\mathrm{Mn}$ and $\mathrm{Pd}$ in $\mathrm{MnPd}_{3}$ film is $28 \%$ and $72 \%$ (data not shown), respectively.

Fig. 1a shows the unit cell of $\mathrm{MnPd}_{3}$. We performed grazing incidence $\theta-2 \theta \mathrm{X}$-ray diffraction (XRD) measurements on a $\mathrm{Si} / \mathrm{SiO}_{2} / \mathrm{MnPd}_{3}(50 \mathrm{~nm})$ sample, as shown in Fig. 1b. For the grazing incidence XRD measurement $\Phi$ and $\Omega$ were fixed at $20^{\circ}$ and $3.5^{\circ}$, respectively. From the intensity of the peaks and pole figures (Supplementary 1 and Fig. S1), its notable that $\mathrm{MnPd}_{3}$ film has a strong (114) texture. The lattice parameters are estimated to be $a=3.89 \AA, b=3.88 \AA$, and $\mathrm{c}=15.42 \AA$ indexed by using ref. $\left({ }^{32}\right)$. The cross-section transmission electron microscopy (TEM) bright image of the MP10 sample is presented in Fig. 1c. The high angle annular dark field (HAADF) image (data not shown) and the bright image both show that the $\mathrm{MnPd}_{3}$ layer grown on thermally oxidized silicon is polycrystalline. The $\mathrm{CoFeB}$ and $\mathrm{MgO}$ layers are also polycrystalline. The electric and magnetotransport measurements were performed on $\mathrm{Si} / \mathrm{SiO}_{2} / \mathrm{MnPd}_{3}(10 \mathrm{~nm}) / \mathrm{MgO}(2 \mathrm{~nm}) / \mathrm{Ta}(2 \mathrm{~nm})$ heterostructure, it will be labelled as $\mathrm{MnPd}(10$ nm) sample (Supplementary 2 and Fig. S2). The resistivity shows metallic behavior coinciding with the possible transition from a paramagnetic to an antiferromagnetic state below $50 \mathrm{~K}$, as shown in Fig. 1d. The ordinary Hall resistance as a function of the external magnetic field is nonlinear at small fields. From the high-field linear region, we estimate a carrier concentration of 4.4 
123 Hall resistance (PHR) are estimated to be $0.012 \%$ and $20 \mathrm{~m} \Omega$ in $\mathrm{MnPd}(10 \mathrm{~nm})$ sample, 124 respectively. The Néel temperature of $\mathrm{MnPd}(10 \mathrm{~nm})$ sample is approximately $37 \mathrm{~K}$ inferred 125 from using temperature-dependent magnetometry (Supplementary 3 and Fig. S3). Polarized 126 neutron reflectometry (PNR) measurements show weak ferromagnetism, persisting upto room 127 temperature, in $\mathrm{MnPd}_{3}$ films possibly originating from uncompensated $\mathrm{Mn}$ moments, Mn 128 clusters, or local ferromagnetic Mn-based compound formation. At room temperature the 129 ferromagnetic component of the magnetization in $\mathrm{MnPd}_{3}$ is determined to be $9 \pm 1.9 \mathrm{kA} / \mathrm{m} \mathrm{using}$ 130 polarized neutron reflection (PNR) whereas at $6 \mathrm{~K}$ it is $\sim 43 \pm 3.4 \mathrm{kA} / \mathrm{m}$ (Supplementary 4 and 131 Fig. S4).
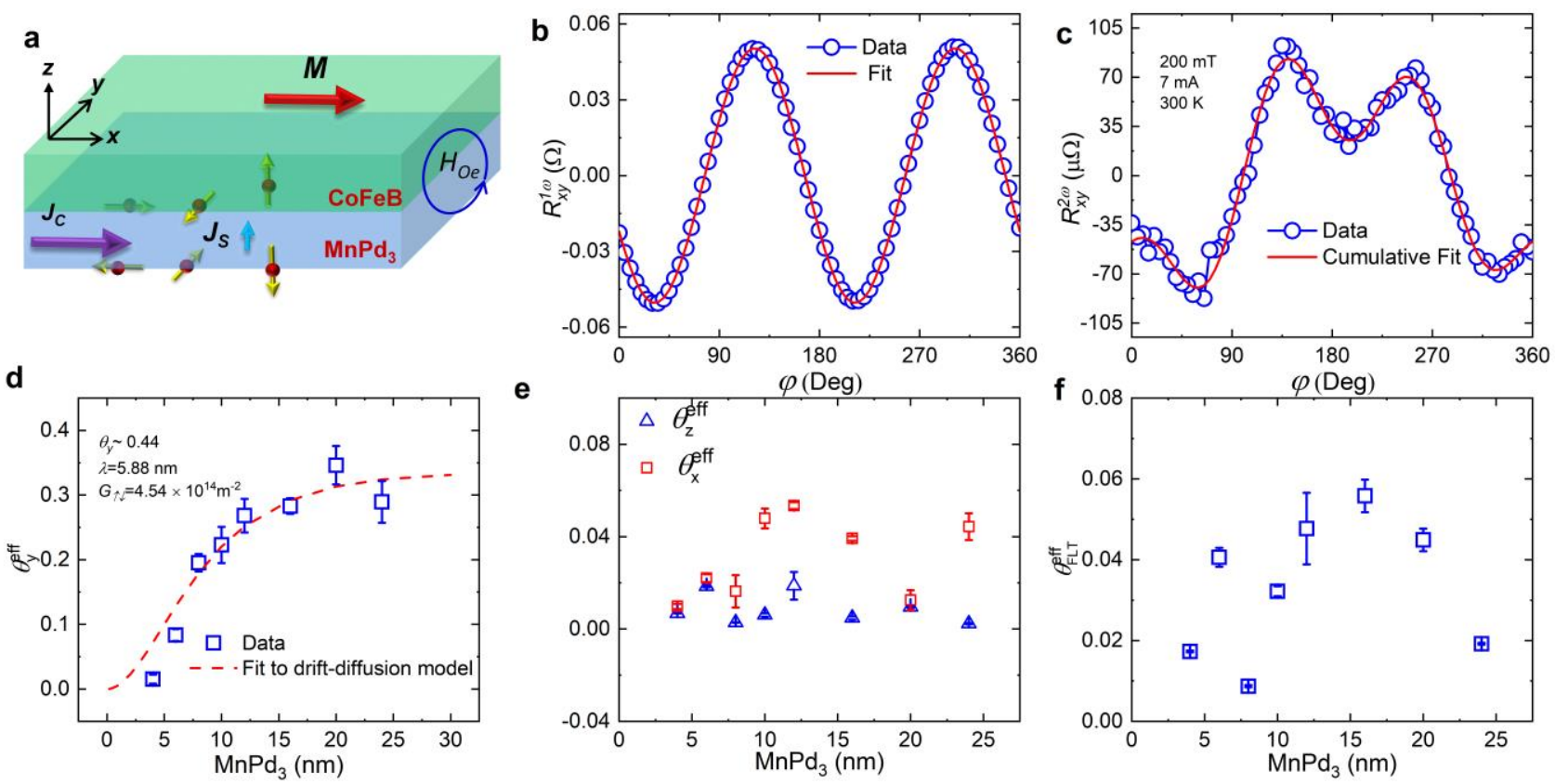

132 $\mathrm{Si} / \mathrm{SiO}_{2} / \mathrm{MnPd}_{3}(\mathrm{x} \mathrm{nm}) / \mathrm{CoFeB}(5 \mathrm{~nm}) / \mathrm{MgO}(2 \mathrm{~nm}) / \mathrm{Ta}(2 \mathrm{~nm}):$ a, Schematic diagram showing in-plane charge current generated spin current with spin polarizations along three axes. The red 
137 spheres represent electrons and yellow arrows represent spin magnetic moment, respectively. b, and $\mathbf{c}, R_{x y}^{1 \omega}$ and $R_{x y}^{2 \omega}$, respectively, as a function of in-plane magnetic field rotation at a fixed amplitude of $200 \mathrm{mT}$ in MP12 sample. d, e, and f, Effective spin torque efficiency due to the inplane anti-damping-like, in-plane and out-of-plane anti-damping-like and field-like torques, 141 respectively, as a function of $\mathrm{MnPd}_{3}$ film thickness. The Hall bar device used for this measurement was $10 \mu \mathrm{m}$ wide and $130 \mu \mathrm{m}$ long, respectively.

We performed SOT measurements using SHH technique on MP4-MP24 samples, control MP (10 nm) and $\mathrm{Si} / \mathrm{SiO}_{2} / \mathrm{CoFeB}(5 \mathrm{~nm}) / \mathrm{MgO}(2 \mathrm{~nm}) / \mathrm{Ta}(2 \mathrm{~nm})$ samples, and a reference $\mathrm{Si} / \mathrm{SiO}_{2} / \mathrm{Pt}(10 \mathrm{~nm}) / \mathrm{CoFeB}(5 \mathrm{~nm}) / \mathrm{MgO}(2 \mathrm{~nm}) / \mathrm{Ta}(2 \mathrm{~nm})$ ( labelled as Pt10 sample) sample ${ }^{33}$. The MP4-MP24 samples and reference sample were patterned into Hall bars with length 130 and 147 width $10 \mu \mathrm{m}$, respectively. The details of the SHH are presented in Supplementary 5 and Fig. S5. 148 The a.c. current injected into the Hall bar induces effective spin-orbit fields, which oscillate the 149 magnetization around its equilibrium position, and as a result, a SHH voltage is induced. In the 150 SHH measurement, the sample is rotated in the $x-y$ plane under constant static magnetic field to 151 keep the magnetization in a single domain state. The spin current with the spin magnetic moment 152 pointing along the negative $y$-axis and positive $x$-and $z$-axes get accumulated between $\mathrm{MnPd}_{3}$ and $153 \mathrm{CoFeB}$ layers as shown in Fig. 2a. Thus, accumulated spin currents exert $\boldsymbol{\tau}_{\boldsymbol{A D L}, \boldsymbol{x}}, \boldsymbol{\tau}_{\boldsymbol{A D L} \boldsymbol{L}, \boldsymbol{y}}$ and $154 \boldsymbol{\tau}_{\boldsymbol{A D L , Z}}, \boldsymbol{\tau}_{\boldsymbol{F L}}$ on the CoFeB layer along the in-plane and out-of-plane directions, respectively. In 155 addition, the Oersted field $\left(H_{O e}\right)$ generated due to the a.c. current flow in the $\mathrm{MnPd}_{3}$ layer exerts 156 an Oersted torque $\left(\boldsymbol{\tau}_{\boldsymbol{O} \boldsymbol{e}}\right)$ on the CoFeB layer. Fig. 2b shows $R_{x y}^{1 \omega}$ as a function of the in-plane 157 magnetic field angle $(\varphi) . R_{x y}^{1 \omega}$ fits perfectly to the $\sin 2 \varphi$, indicating that the out-of-plane field 158 projection due to the imperfect sample mounting is absent. $R_{x y}^{2 \omega}$ as a function of magnetic field 159 angle is presented in Fig. 3c. Since the torques have different dependencies with $\varphi$, we can 
160 extract $R_{x y}^{2 \omega}$ due to different types of torques using SHH. In MP10 sample, the extracted spin161 orbit fields associated with the $\boldsymbol{\tau}_{\boldsymbol{A D L}, \boldsymbol{x}}, \boldsymbol{\tau}_{\boldsymbol{A D L}, \boldsymbol{y}}, \boldsymbol{\tau}_{\boldsymbol{A D L}, \boldsymbol{Z}}$, and $\boldsymbol{\tau}_{\boldsymbol{F} L}$ are $(0.02 \pm 0.002),(0.132 \pm$ 162 0.002), $(0.0036 \pm 0.0003)$, and $(0.019 \pm 0.00) \mathrm{mT}$ per $10^{6} \mathrm{~A} / \mathrm{cm}^{2}$, respectively. These values of 163 the $\boldsymbol{\tau}_{\boldsymbol{A D L}, \boldsymbol{y}}$ and $\boldsymbol{\tau}_{\boldsymbol{F} \boldsymbol{L}}$ are comparable or better than the previous reports on heavy 164 metals/ferromagnet ${ }^{33}$, TIs/ferromagnet ${ }^{11,12}$, and Weyl semimetal/ferromagnets ${ }^{19}$. In Fig. 2d $165 \theta_{y}^{\text {eff }}$ as a function of $\mathrm{MnPd}_{3}$ film thickness is presented, which shows heavy metal like behavior. 166 The simple drift-diffusion model (Eqn. 1) can be utilized to extract bulk spin-torque efficiency 167 figure of merit $\left(\theta_{y}(t \approx \infty)\right)$ and spin-diffusion length $(\lambda)$,

$168 \theta_{y}^{e f f}=\theta_{y}(t \approx \infty)\left(1-\operatorname{sech}\left(\frac{t}{\lambda}\right)\right)$

169 where $t$ is $\mathrm{MnPd}_{3}$ film thickness. This drift-diffusion model considers that the spin current 170 generated by the bulk of thin films is completely absorbed by the ferromagnetic layer without 171 any dissipation at the interface and back-flow of the spin current. $\theta_{y}(t \approx \infty)$ and $\lambda$ obtained by 172 ordinary drift-diffusion model are 0.34 and $6.30 \mathrm{~nm}$, respectively. Now by considering spin-back 173 flow the drift-diffusion model can be modified into ${ }^{34-36}$ :

$174 \quad \theta_{y}^{e f f}=\theta_{y}(t \approx \infty)\left(1-\operatorname{sech}\left(\frac{t}{\lambda}\right)\right)\left[\frac{1+\tanh \left(\left(\frac{t}{2 \lambda}\right)\right.}{2 \rho \lambda G_{\uparrow \downarrow}}\right]^{-1}$

175 where $\rho$ is bulk resistivity, $G_{\uparrow \downarrow}$ is spin-mixing conductivity, respectively. The red line in Fig. $2 \mathrm{~d}$ 176 is a fit to Eqn. (2) with $\theta_{y}(t \approx \infty)$ and $\lambda$ as independent fitting parameters. $G_{\uparrow \downarrow}$ values of MP4 177 and MP24 samples are estimated to be $4.54 \times 10^{14} \mathrm{~m}^{-2}$ and $3.70 \times 10^{15} \mathrm{~m}^{-2}$, respectively. The 178 extracted values of $\theta_{y}(t \approx \infty)$ and $\lambda$ are 0.44 and $5.88 \mathrm{~nm}$, respectively for $G_{\uparrow \downarrow}$ value of $4.54 \times$ $17910^{14} \mathrm{~m}^{-2} . \rho$ value used for the fitting was $60 \mu \Omega \mathrm{cm}$ obtained by measuring four terminal 
180

181

182

183

184

185

186

187

188

189

190

191

192

193

194

195

196

197

198

199

200

resistance of $\mathrm{Sub} / \mathrm{MnPd}_{3}(20 \mathrm{~nm}) / \mathrm{MgO}(2 \mathrm{~nm}) / \mathrm{Ta}(2 \mathrm{~nm})$ sample. The extracted values of $\theta_{y}(t \approx \infty)$ and $\lambda$ are 0.36 and $6.30 \mathrm{~nm}$, respectively for $G_{\uparrow \downarrow}$ value of $3.70 \times 10^{15} \mathrm{~m}^{-2}$. The values of the $\sigma_{z x}^{y}$ corresponding to $\theta_{y}(0.34-0.44)$ are estimated to be $(5.67-7.33) \times 10^{5} \hbar / 2 e \Omega^{-1} \mathrm{~m}^{-1}$. These values of $\sigma_{z x}^{y}$ are among the largest for the reported values of the antiferromagnets ${ }^{15,37}$, heavy metals ${ }^{6,38,39}$, topological insulators ${ }^{8,11}$, and Weyl semimetals ${ }^{19} \cdot R_{x y}^{2 \omega}$ in the control samples does not show any field or $\varphi$ dependence indicating that the SOTs in the MP samples originates from the $\mathrm{MnPd}_{3}$ layer (Supplementary 5 and Figs. S5 and S6). The values for the spin-orbit fields associated with $\boldsymbol{\tau}_{\boldsymbol{A D L}, \boldsymbol{y}}$ and $\boldsymbol{\tau}_{\boldsymbol{F} \boldsymbol{L}}$ of the reference Pt10 sample are estimated to be $(0.040 \pm 0.003)$ and $(0.012 \pm 0.001) \mathrm{mT}$ per $10^{6} \mathrm{~A} / \mathrm{cm}^{2}$, respectively (Supplementary 7 and Fig. S7). The estimated value of $\theta_{y}^{\text {eff }}$ and $\theta_{F L}^{\text {eff }}$ are $(0.07 \pm 0.01)$ and $(0.02 \pm 0.002)$, respectively in agreement with the previous reports ${ }^{6,33,40}$. After post annealing at $400{ }^{\circ} \mathrm{C}$, the reference $\mathrm{Pt} 10$ sample does not show a SHH signal, suggesting that its SOT did not withstand such annealing.

As presented in Fig. 2e, $\theta_{z}^{\text {eff }}$ and $\theta_{x}^{\text {eff }}$ do not depend on the $\mathrm{MnPd}_{3}$ film thickness as $\theta_{y}^{\text {eff }}$ does. $\theta_{F L}^{\text {eff }}$ shown in Fig. $2 \mathrm{~d}$ also does not show any specific $\mathrm{MnPd}_{3}$ film thickness dependence. The $\sigma_{z x}^{z, e f f}$ value in MP12 sample is as large as $\sim 0.14 \times 10^{5} \hbar / 2 e \Omega^{-1} \mathrm{~m}^{-1} . \sigma_{z x}^{z, e f f}$ in MP samples is comparable or better than recent reports on $\mathrm{WTe}_{2} / \mathrm{Py}^{18}$ and $\mathrm{Mn}_{3} \mathrm{GaN} / \mathrm{Py}{ }^{17} \cdot \sigma_{z x}^{x, e f f}$ in MP12 sample is $0.77 \times 10^{5} \hbar / 2 e \Omega^{-1} \mathrm{~m}^{-1}$. These values of $\theta_{k}$ and $\sigma_{i j}^{k}$ are largest among the reported values as listed in Table 1 . The difference in the thickness dependence of the $\theta_{x, y, z}^{e f f}$ indicate that their origins are also different.

We also performed ST-FMR measurement on MP10 sample as a confirmation of the observed SOT with SHH technique. The details of ST-FMR are presented in Supplementary 8 
201 and Fig. S8. $\theta_{y}^{\text {eff }}$ estimated by using ST-FMR of MP10 sample is $(0.21 \pm 0.01)$ whereas it is $202(0.22 \pm 0.03)$ estimated using $\mathrm{SHH}$. We also prepared reference samples $\mathrm{Si} / \mathrm{SiO}_{2} / \mathrm{Pt}(6$ and 10 $203 \mathrm{~nm}) / \mathrm{CoFeB}(5 \mathrm{~nm}) / \mathrm{MgO}(2 \mathrm{~nm}) / \mathrm{Ta}(2 \mathrm{~nm})$ (labelled as Pt6 and Pt10 samples) and $\mathrm{Si} / \mathrm{SiO}_{2} / \mathrm{W}(6$ $204 \mathrm{~nm}) / \mathrm{CoFeB}(2 \mathrm{~nm}) / \mathrm{MgO}(2 \mathrm{~nm}) / \mathrm{Ta}(2 \mathrm{~nm})$ (labelled as $\mathrm{W}$ sample) for the ST-FMR 205 measurements. $\theta_{y}^{e f f}$ of as deposited Pt samples is $0.06 \pm 0.01$ and $0.08 \pm 0.01$, respectively 206 (Supplementary 9 and Fig. S9). However, after post annealing at $400{ }^{\circ} \mathrm{C}$ the reference Pt samples 207 do not show a ST-FMR signal, suggesting that SOT did not withstand the annealing process. $208 \theta_{y}^{e f f}$ of as deposited W sample is determined to be $-0.43 \pm 0.03$ at $6 \mathrm{GHz}$ excitation frequency. 209 The estimated $\sigma_{z x}^{y, \text { eff }}$ is $-1.43 \times 10^{5} \hbar / 2 e \Omega^{-1} \mathrm{~m}^{-1}$. This value of $\theta_{y}^{\text {eff }}$ of the as deposited W 210 sample is comparable to the previous report ${ }^{41} \cdot \theta_{y}^{\text {eff }}$ of the $\mathrm{W}$ sample post annealed at $400{ }^{\circ} \mathrm{C}$ for 21130 minutes is estimated to be -0.011 and the corresponding $\sigma_{y}^{\text {eff }}$ is $-0.13 \times 10^{5} \hbar / 2 e \Omega^{-1} \mathrm{~m}^{-1}$ 212 (Supplementary 9 and Fig. S9). 


\begin{tabular}{|c|c|c|c|c|c|c|c|c|c|c|}
\hline Materials & $\begin{array}{l}\rho \\
(\mu \Omega \mathrm{cm})\end{array}$ & $\theta_{x}^{\text {eff }}$ & $\theta_{y}$ & $\theta_{z}^{e f f}$ & $\theta_{F L}^{\text {eff }}$ & $\begin{array}{l}\sigma_{z x}^{y}(\hbar / 2 e \\
\left.10^{5} \Omega^{-1} \mathrm{~m}^{-1}\right)\end{array}$ & $\begin{array}{l}J_{s w} \\
\left(\mathrm{MA} / \mathrm{cm}^{2}\right)\end{array}$ & $\begin{array}{l}\text { Switched } \\
\text { magnetic } \\
\text { anisotropy }\end{array}$ & $\begin{array}{l}H_{x} \\
(\mathrm{mT})\end{array}$ & $\begin{array}{l}\text { Post } \\
\text { annealing } \\
\text { temp }\left({ }^{\circ} \mathrm{C}\right)\end{array}$ \\
\hline This work & $60-95$ & 0.053 & $0.34-0.44$ & 0.018 & 0.06 & $5.7-7.3$ & $11-24.7$ & IMA-PMA & Zero & 400 \\
\hline $\mathrm{Pt}^{40}$ & 40 & - & 0.14 & - & 0.08 & 3.5 & 50 & PMA & 70 & As dep. \\
\hline $\mathrm{W}^{38}$ & 188 & - & -0.22 & - & -0.022 & -1.2 & $11^{42}$ & PMA & 20 & $200^{42}-300^{38}$ \\
\hline $\mathrm{MnGaN}^{17}$ & 225 & -0.013 & 0.025 & 0.018 & -0.15 & 0.11 & - & - & - & As dep. \\
\hline $\mathrm{WTe}_{2}{ }^{18}$ & 380 & - & 0.03 & 0.013 & 0.034 & 0.08 & - & - & - & As dep. \\
\hline
\end{tabular}

216 Table 1: Summary of $\theta_{k}, \sigma_{i j}^{k}$, and $\rho$ of different spin channels post annealed at different

217 temperatures and measured at room temperature. 

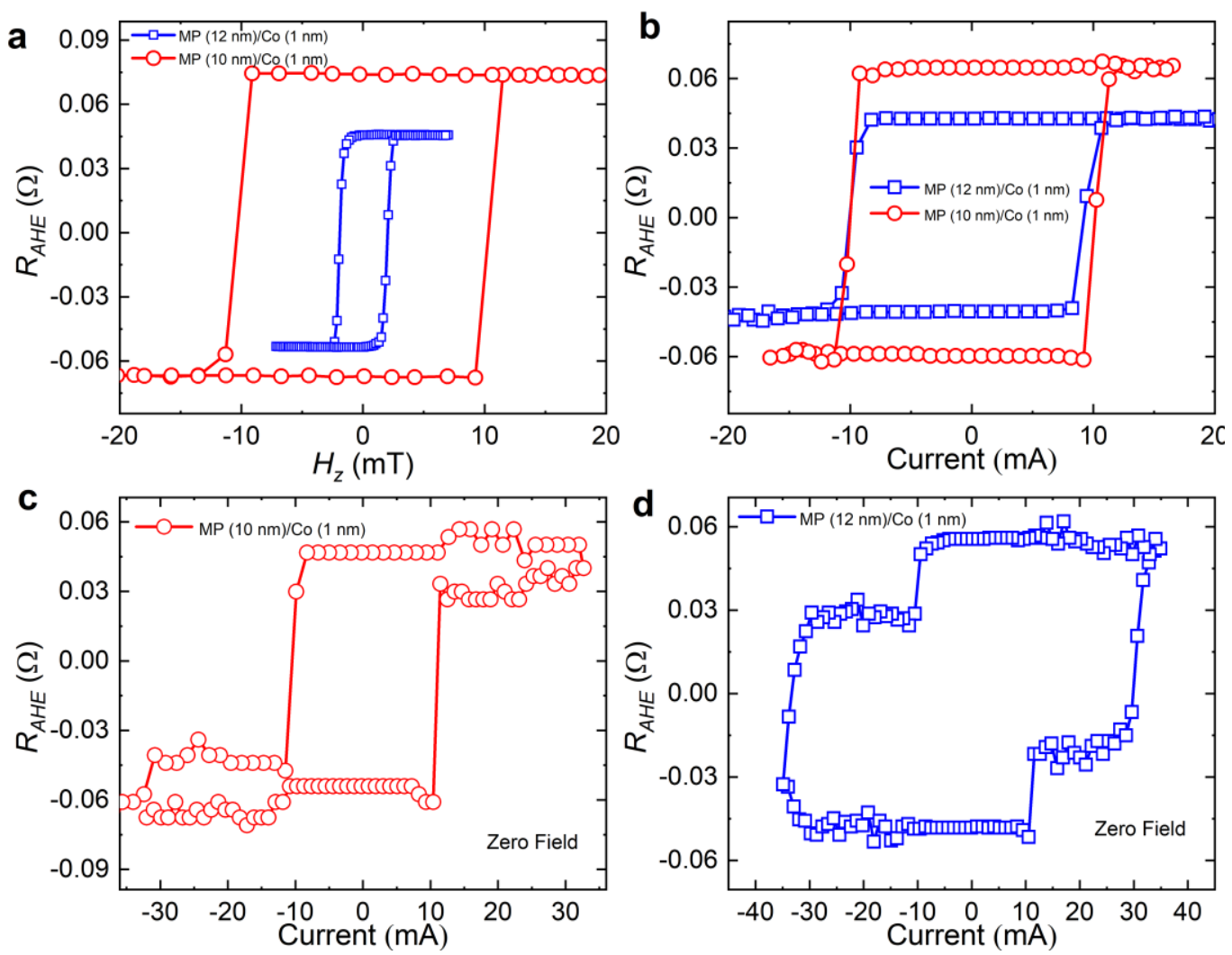

219 Fig. 3. Demonstration of external magnetic field-free out-of-plane magnetization switching

220 via $z$-spin polarization generated anti-damping spin-orbit torque: a, The anomalous Hall

221 resistance as a function of out-of-plane external field. b, Switching of perpendicular Co layer via

222 SOT under the application of $-8 \mathrm{mT}$ and $-20 \mathrm{mT}$ along $x$-direction for MP $(10 \mathrm{~nm}) / \mathrm{Co}(1 \mathrm{~nm})$ and

$223 \mathrm{MP}(12 \mathrm{~nm}) / \mathrm{Co}(1 \mathrm{~nm})$ samples, respectively. c, and d, Field-free perpendicular Co layer

224 switching via out-of-plane anti-damping-like torque generated by $z$-spin polarization. The Hall

225 bar with a length of $130 \mu \mathrm{m}$ and a width of $10 \mu \mathrm{m}$ was used for the magnetization switching 226 experiment.

In order to demonstrate external magnetic field-free PMA switching, we prepared 
${ }^{\circ} \mathrm{C}$ for 30 minutes in vacuum and subsequently field-cooled under the application of an out-ofplane magnetic field of $0.45 \mathrm{~T}$. Fig. 3a shows anomalous Hall resistance $\left(R_{A H E}\right)$ as a function of out-of-plane magnetic field. The hysteretic $R_{A H E}$ loop confirms PMA is present in the Co layer. Alternatively, magnetometry was also used to confirm that PMA is present in MP10/Co1 sample (Supplementary 3 and Fig. S3b). Fig. 3b shows current-induced SOT magnetization switching under the presence of negative external magnetic fields. The write d.c. current pulse width used for PMA switching is $20 \mathrm{~ms}$, which is followed by a read current of $0.4 \mathrm{~mA}$. The full switching of magnetization occurs in MP10/Co1 and MP12/Co1 samples at $10.1 \mathrm{~mA}$ and $9.5 \mathrm{~mA}$, respectively. Then, in the absence of external magnetic field, the d.c. current in pulses is swept from $-36 \mathrm{~mA}$ to $+36 \mathrm{~mA}$ with a step size of $1.06 \mathrm{~mA}$. For MP10/Co1 as shown in Fig. 3c, partial switching of the magnetization occurs at a positive current of about $10.78 \mathrm{~mA}$, and complete switching occurs at $\sim 32.4 \mathrm{~mA}\left(\sim 37.0 \mathrm{MA} / \mathrm{cm}^{2}\right)$. In the subsequent reverse sweep partial switching of magnetization occurs at $\sim-10.87 \mathrm{~mA}$ and complete switching occurs at $\sim 31.63$ mA. For MP12/Co1 sample as shown in Fig. 3d, partial switching of magnetization occurs at a positive current of $~ 11.35 \mathrm{~mA}$. Continuously sweeping the d.c. pulses switches the remaining magnetization at $\sim 30.92 \mathrm{~mA}\left(\sim 24.7 \mathrm{MA} / \mathrm{cm}^{2}\right)$. Subsequently reverse sweeping the current pulses, partial switching of magnetization occurs at $~-9.60 \mathrm{~mA}$ and switching of remaining magnetization occurs at $\sim-33.32 \mathrm{~mA}$. Since the $R_{A H E}$ values obtained by field sweep and current sweep are close, we can conclude that the full switching of PMA has been observed in both PMA samples. $J_{S w}$ values observed in our PMA samples without external magnetic field is comparable or better than the previously reported values in $\mathrm{Pt} / \mathrm{Co}^{5,6}\left(\sim 23-100 \mathrm{MA} / \mathrm{cm}^{2}\right), \mathrm{Pd}_{0.25} \mathrm{Pt}_{0.75} / \mathrm{Co}^{43}$ $\left(\sim 22 \mathrm{MA} / \mathrm{cm}^{2}\right)$, Pt/antiferromagnet ${ }^{44}$ with external magnetic field. The SOT switching of magnetization in our PMA samples results from the interplay of $\boldsymbol{\tau}_{\boldsymbol{A D L}, \boldsymbol{x}}, \boldsymbol{\tau}_{\boldsymbol{A D L}, \boldsymbol{y}}, \boldsymbol{\tau}_{\boldsymbol{A D L}, \boldsymbol{z}}$, and $\boldsymbol{\tau}_{\boldsymbol{F L} \boldsymbol{L}}$. 
253 In the presence of external magnetic field the $R_{A H E}$ vs $I$ loop shows similar behavior as that of 254 positive $\theta_{y}^{e f f}$ such as in the case of $\mathrm{Pt} / \mathrm{Co} / \mathrm{AlOx}^{5,6}$. In the absence of an external magnetic field, if 255 there is only $\boldsymbol{\tau}_{\boldsymbol{A D L}, \boldsymbol{Z}}$, PMA switching occurs via anti-damping process, which is confirmed by 256 numerically solving Landau-Lifshitz-Gilbert (LLG) equation (Supplementay 10 and Fig. S10b). 257 In the presence of a relatively weaker $\boldsymbol{\tau}_{\boldsymbol{A D L , z}, \boldsymbol{B}}$ and $\boldsymbol{\tau}_{\boldsymbol{A D L}, \boldsymbol{x}}$ and strong $\boldsymbol{\tau}_{\boldsymbol{A D L}, \boldsymbol{y}}$ the magnetization is 258 partially switched at a lower current $(\sim 10 \mathrm{~mA})$, which results in an intermediate state. The 259 intermediate state can occur due to an insufficient external magnetic field, which is unable to 260 completely break mirror symmetry ${ }^{45}$. Previously, intermediate magnetic states were observed 261 below threshold $J_{s w}$ (Ref. ${ }^{46}$ ). The external magnetic field-free switching of PMA 262 unambiguously demonstrates the presence of $\hat{\sigma}_{z}$ generated $\boldsymbol{\tau}_{\boldsymbol{A D L}, \boldsymbol{z}}$ in the MP samples. These 263 experimental results of PMA switching have been qualitatively reproduced by the LLG 264 simulations (Supplementary 10 and Figs. S10c and S10d). The intermediate states observed 265 could be utilized for neuromorphic computing ${ }^{13}$. In addition to field-free PMA switching, we 266 also performed field-free magnetization switching of the in-plane CoFeB layer in MP24 sample, 267 as detected by using unidirectional spin Hall magnetoresistance (USMR) mechanism ${ }^{47,48}$ 268 (Supplementary 11 and Fig. S11). $J_{s w}$ is estimated to be $\sim 11.0 \mathrm{MA} / \mathrm{cm}^{2}$ using the parallel resistor 269 model.

We also performed SHH measurements on the PMA samples, as detailed in

271 Supplementary 13 and Fig. s13. The magnitude of the SHH resistance $\left(R_{x y}^{2 \omega}\right)$ is not symmetric at 272 up and down magnetizations when the field is swept along $y$-axis. If there were only $\boldsymbol{\tau}_{\boldsymbol{F L}}$ and $\boldsymbol{\tau}_{\boldsymbol{O} \boldsymbol{e}}$ 273 present in MP samples, the magnitude and field dependence of $R_{x y}^{2 \omega}$ would remain the same since 274 the spin-orbit field associated to them is independent of the magnetization polarity as in the case 275 of $\mathrm{Ta} / \mathrm{CoFeB} / \mathrm{MgO}^{23}$. The spin-orbit field associated with $\boldsymbol{\tau}_{\boldsymbol{A D L}, \boldsymbol{x}}\left(\boldsymbol{H}_{\boldsymbol{A D L}, \boldsymbol{x}} \sim\left(\hat{\sigma}_{\boldsymbol{x}} \times \widehat{m}\right)\right)$ switches 
276 sign as the magnetization switches sign, which results in the unequal and different field 277 dependence of $R_{x y}^{2 \omega}$. This clearly shows the presence of torque generated by $\hat{\sigma}_{x}$ in MP samples. $\theta_{x}^{e f f}, \theta_{y}^{e f f}$, and $\theta_{F L}^{e f f}$ of the MP10/Co1 sample (MP12/Co1 sample) are $0.023 \pm 0.001(0.040 \pm$ $0.003), 0.24 \pm 0.02(0.32 \pm 0.03)$, and $0.03 \pm 0.001(0.12 \pm 0.01)$, respectively. These values are comparable to the SHH-measured SOT efficiencies of IMA MP10 and MP12 samples.
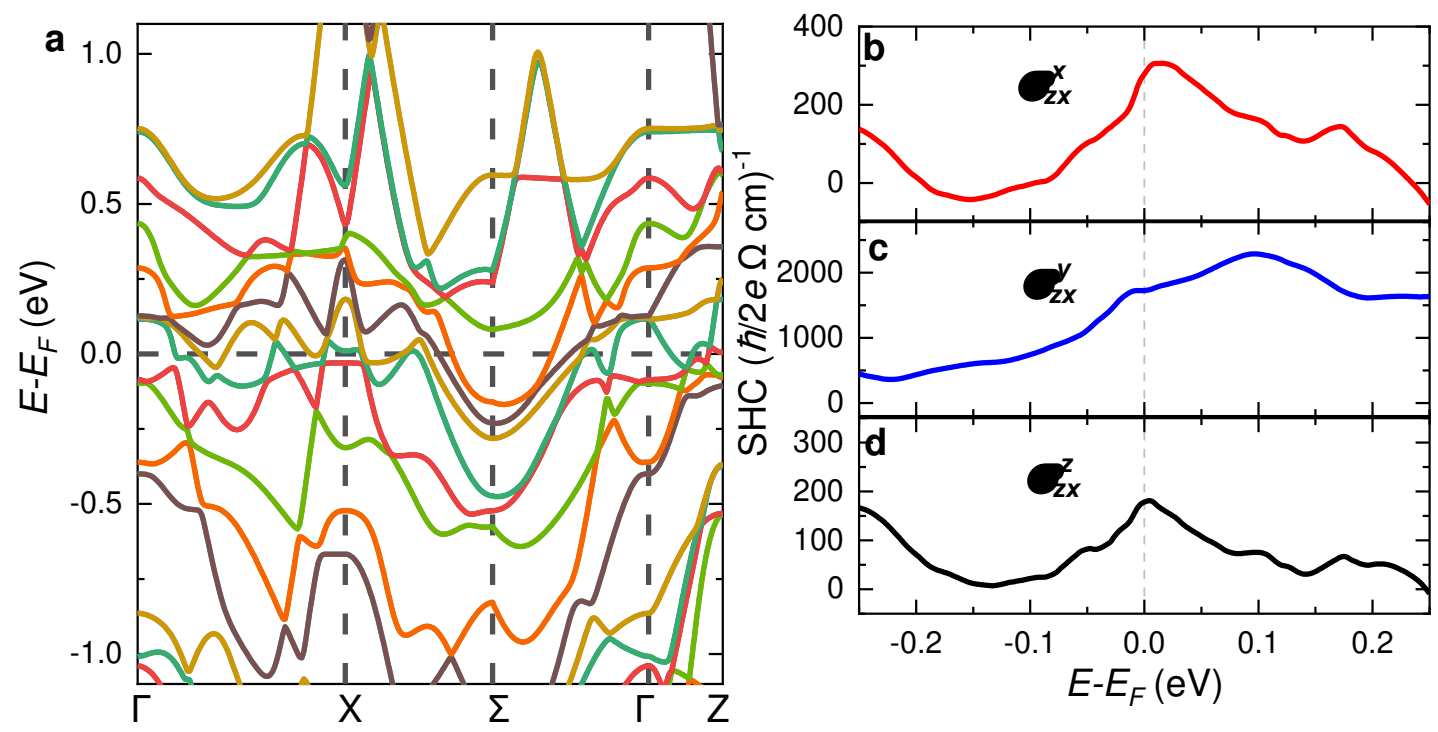

Fig. 4: Effect of (114) texture on the spin polarization. a, The calculated band structure of stoichiometric $\mathrm{MnPd}_{3}$ at room temperature. b, $\mathbf{c}$, and $\mathbf{d}$, The calculated $\sigma_{z x}^{x}, \sigma_{z x}^{y}$, and $\sigma_{z x}^{z}$ as a function of energy for $\operatorname{MnPd}_{3}$ (114) film, where the $x$ axis is oriented along the [4 01$]$ direction.

We explain the appearance of the non-vanishing $\hat{\sigma}_{x}$ and $\hat{\sigma}_{z}$ in terms the contribution from grains of different orientations in our polycrystalline $\mathrm{MnPd}_{3}$ films. $\mathrm{MnPd}_{3}$ has crystal space 287 group I4/mmm $\left[{ }^{32}\right]$. If the film was monocrystalline and (001) oriented with the charge current 288 flowing along the [100] direction (the $x$-direction), only the conventional spin Hall conductivity $289 \sigma_{z x}^{y}$ (where the spin current flows along the [001] direction (the $z$-direction) normal to the charge current and has spin polarization along the [010] direction (the $y$-direction)), would be allowed 
291 due to the (001) plane being invariant to all symmetry operations of this space group. In a 292 polycrystalline film, however, other crystal orientations with lower symmetries contribute to the 293 spin Hall conductivity. For example, the (114) plane corresponding to the dominant texture of 294 our films (reflected by the strongest XRD peak in Fig. 1 (b)) is only invariant with respect to 295 mirror symmetry $M_{[\overline{1} 10]}$ and two-fold rotation $C_{[\overline{1} 10]}$. This allows the appearance of 296 unconventional components of the spin Hall conductivity tensor, such as $\sigma_{z x}^{x}$ and $\sigma_{z x}^{Z}$, where the 297 spin polarization is parallel to the direction of the spin current or the charge current. Moreover, in 298 a polycrystalline film, the current direction itself varies with respect to the high symmetry 299 directions of different grains, which also influences the shape of the spin Hall conductivity 300 tensor. These aspects have been discussed in Ref. $\left[{ }^{15}\right]$.

301 To quantitatively evaluate the contribution from this mechanism, we perform first-principles 302 density functional theory calculations of spin Hall conductivity of bulk $\mathrm{MnPd}_{3}$ assuming a room303 temperature paramagnetic phase. Figure 4a shows the calculated band structure of $\mathrm{MnPd}_{3}$. There 304 are several bands crossing the Fermi level $\left(E_{F}\right)$, indicating the metallic ground state. The small 305 gaps between the bands near $E_{F}$ are favorable for the sizable spin Hall conductivity ${ }^{49}$, which is 306 given by ${ }^{50}$

$307 \quad \sigma_{i j}^{k}=\frac{e^{2}}{\hbar} \int \frac{d^{3} \vec{k}}{(2 \pi)^{3}} \sum_{n} f_{n \vec{k}} \Omega_{n, i j}^{k}(\vec{k})$,

$308 \Omega_{n, i j}^{k}(\vec{k})=-2 \operatorname{Im} \sum_{n^{\prime} \neq n} \frac{\left\langle n \vec{k}\left|J_{i}^{k}\right| n^{\prime} \vec{k}\right|\left\langle n^{\prime} \vec{k}\left|v_{j}\right| n \vec{k}\right\rangle}{\left(E_{n \vec{k}}-E_{n^{\prime} \vec{k}}\right)^{2}}$,

309 where $f_{n \vec{k}}$ is the Fermi-Dirac distribution function for band $n$ and wave vector $\vec{k}, \Omega_{n, i j}^{k}(\vec{k})$ is the 310 spin Berry curvature, $J_{i}^{k}=\frac{1}{2}\left\{v_{i}, s_{k}\right\}$ is the spin current operator, $v_{i}$ and $s_{k}$ are velocity and spin 311 operators, respectively, and $i, j, k=x, y, z$. As expected, for $\operatorname{MnPd}_{3}$ textured in the (001) plane, 
312 only the conventional $\sigma_{z x}^{y}$ is non-vanishing (Table 2 in Supplementary 13). However, for the 313 dominant (114) stacking texture, the unconventional spin Hall conductivities $\left(\sigma_{z x}^{x}\right.$ and $\left.\sigma_{z x}^{Z}\right)$ 314 emerge (Table 2 in Supplementary 13). Figures 4b-d show the calculated spin Hall 315 conductivities for $\mathrm{MnPd}_{3}$ (114) film as a function of energy when the charge current flows along 316 the $[\overline{4} 01]$ direction (the $x$-direction). We find a high conventional $\sigma_{z x}^{y} \sim 1744\left(\frac{\hbar}{2 e}\right)(\Omega \mathrm{cm})^{-1}$ and 317 sizable unconventional conductivities $\sigma_{z x}^{x} \sim 279\left(\frac{\hbar}{2 e}\right)(\Omega \mathrm{cm})^{-1}$ and $\sigma_{z x}^{z} \sim 166\left(\frac{\hbar}{2 e}\right)(\Omega \mathrm{cm})^{-1}$ at 318 the Fermi energy. It is evident that the $\sigma_{z x}^{x}$ and $\sigma_{z x}^{z}$ values are approximately an order of 319 magnitude smaller than $\sigma_{z x}^{y}$, which is consistent with our experimental observation. Similarly, 320 other $\mathrm{MnPd}_{3}$ grains with different orientations can also contribute to the unconventional spin 321 Hall conductivity. In summary, we studied anti-damping spin-orbit torques generated by the $\hat{\sigma}_{x}, \hat{\sigma}_{y}$, and $\hat{\sigma}_{z}$ 323 in $\mathrm{MnPd}_{3} /$ ferromagnet heterostructure. At least two independent characterizations were 324 performed to verify the presence of torques. DFT simulations confirmed the low crystal 325 symmetry present in the (114) oriented MnPd3 thin films as the origin of the observed 326 unconventional SOTs. We demonstrated successful growth of conductive $\mathrm{MnPd}_{3}$ thin films with 327 high $\sigma_{z x}^{x}, \sigma_{z x}^{y}$, and $\sigma_{z x}^{z}$ after post annealing at $400{ }^{\circ} \mathrm{C}$ for half an hour. Complete external 328 magnetic field-free switching of both IMA and PMA were realized. The observed SOTs were 329 robust against thermal treatment, and compatible with low damping constant of $\mathrm{CoFeB}$ even 330 after post annealing. All of these are key factors for the integration of a practical spin current 331 source based on $\mathrm{MnPd}_{3}$ into next generation of SOT-based spintronics devices. 


\section{References}

1. Dieny, B. et al. Opportunities and challenges for spintronics in the microelectronics industry. Nat. Electron. 3, 446-459 (2020).

2. Grimaldi, E. et al. Single-shot dynamics of spin-orbit torque and spin transfer torque switching in three-terminal magnetic tunnel junctions. Nat. Nanotechnol. 15, 111-117 (2020).

3. Manchon, A. et al. Current-induced spin-orbit torques in ferromagnetic and antiferromagnetic systems. Rev. Mod. Phys. 91, 035004 (2019).

4. Chernyshov, A. et al. Evidence for reversible control of magnetization in a ferromagnetic material via spin-orbit magnetic field. Nat. Phys. 5, 656 (2008).

5. Miron, I. M. et al. Perpendicular switching of a single ferromagnetic layer induced by inplane current injection. Nature 476, 189-193 (2011).

6. Liu, L., Lee, O. J., Gudmundsen, T. J., Ralph, D. C. \& Buhrman, R. A. Current-induced switching of perpendicularly magnetized magnetic layers using spin torque from the spin hall effect. Phys. Rev. Lett. 109, 096602 (2012).

7. Fukami, S., Anekawa, T., Zhang, C. \& Ohno, H. A spin-orbit torque switching scheme with collinear magnetic easy axis and current configuration. Nat. Nanotechnol. 11, 621626 (2016).

8. Mellnik, A. R. et al. Spin-transfer torque generated by a topological insulator. Nature 511, 449-451 (2014).

9. Fan, Y. et al. Magnetization switching through giant spin-orbit torque in a magnetically 
doped topological insulator heterostructure. Nat. Mater. 13, 699-704 (2014).

355 10. Kondou, K. et al. Fermi level dependent charge-to-spin current conversion by Dirac 356 surface state of topological insulators. Nat. Phys. 12, 1027-1032 (2016).

357 11. DC, M. et al. Room-temperature high spin-orbit torque due to quantum confinement in sputtered $\mathrm{Bi}_{\mathrm{x}} \mathrm{Se}_{(1-\mathrm{x})}$ films. Nat. Mater. 17, 800-807 (2018).

12. Khang, N., Khang, D., Ueda, Y. \& Hai, P. N. A conductive topological insulator with 360

361

13. Fukami, S., Zhang, C., DuttaGupta, S., Kurenkov, A. \& Ohno, H. Magnetization switching by spin-orbit torque in an antiferromagnet-ferromagnet bilayer system. Nat. Mater. 15, 535-541 (2016).

14. Oh, Y. W. et al. Field-free switching of perpendicular magnetization through spin-orbit torque in antiferromagnet/ferromagnet/oxide structures. Nat. Nanotechnol. 11, 878-884 (2016).

15. Zhang, W. et al. Giant facet-dependent spin-orbit torque and spin Hall conductivity in the triangular antiferromagnet $\mathrm{IrMn}_{3} . S c i . A d v .2$, (2016).

16. Chen, J.-Y. et al. Field-free spin-orbit torque switching of composite perpendicular $\mathrm{CoFeB} / \mathrm{Gd} / \mathrm{CoFeB}$ layers utilized for three-terminal magnetic tunnel junctions. Appl. Phys. 
375 18. Macneill, D. et al. Control of spin-orbit torques through crystal symmetry in $\mathrm{WTe}_{2}$

376 /ferromagnet bilayers. Nat. Phys. 13, 300-306 (2017).

377 19. Shi, S. et al. All-electric magnetization switching and Dzyaloshinskii-Moriya interaction in WTe2/ferromagnet heterostructures. Nat. Nanotechnol. 14, 945-949 (2019).

379 20. Song, P. et al. Coexistence of large conventional and planar spin Hall effect with long spin 380 diffusion length in a low-symmetry semimetal at room temperature. Nat. Mater. 19, 292$381298(2020)$.

382 21. Qi, X.-L. \& Zhang, S.-C. Topological insulators and superconductors. Rev. Mod. Phys. 83, $383 \quad 1057-1106(2011)$.

384 22. Zhao, B. et al. Unconventional charge-spin conversion in weyl-semimetal WTe2. Adv. $385 \quad$ Mater. 2000818 (2020).

386 23. Baek, S. C. et al. Spin currents and spin-orbit torques in ferromagnetic trilayers. Nat. $387 \quad$ Mater. 17, 509-513 (2018).

388 24. Lee, D. \& Lee, K. Spin-orbit Torque Switching of Perpendicular Magnetization in 389 Ferromagnetic Trilayers. Sci. Rep. 10, 1772 (2020).

390 25. Li, X. et al. Materials Requirements of High-Speed and Low-Power Spin-Orbit-Torque 391 Magnetic Random-Access Memory. IEEE J. Electron Devices Soc. 8, 674 - 680 (2020).

392 26. Mangin, S. et al. Current-induced magnetization reversal in nanopillars with perpendicular 393 anisotropy. Nat. Mater. 5, 210-215 (2006).

394 27. Han, J. et al. Room-temperature spin-orbit torque switching induced by a topological 
insulator. Phys. Rev. Lett. 119, 077702 (2017).

396 28. Li, P. et al. Magnetization switching using topological surface states. Sci. Adv. 5, (2019).

397 29. Zhao, Z., Smith, A. K., Jamali, M. \& Wang, J. P. External-field-free spin Hall switching 398 of perpendicular magnetic nanopillar with a dipole-coupled composite structure. $A d v$. Electron. Mater. 6, 1901368 (2020).

400

401

402

403

404

405

406

407

408

409

410

411

412

413 414
30. Krizakova, V., Garello, K., Grimaldi, E., Kar, G. S. \& Gambardella, P. Field-free switching of magnetic tunnel junctions driven by spin-orbit torques at sub-ns timescales. Appl. Phys. Lett. 116, 232406 (2020).

31. Wang, M. et al. Field-free switching of a perpendicular magnetic tunnel junction through the interplay of spin-orbit and spin-transfer torques. Nat. Electron. 1, 582-588 (2018).

32. Coldea, M. et al. X-ray photoelectron spectroscopy and magnetism of Mn-Pd alloys. $J$. Alloys Compd. 417, 7-12 (2006).

33. Avci, C. O. et al. Interplay of spin-orbit torque and thermoelectric effects in ferromagnet/normal-metal bilayers. Phys. Rev. B 90, 224427 (2014).

34. Chen, Y.-T. et al. Theory of spin Hall magnetoresistance. Phys. Rev. B 87, 144411 (2013).

35. Zhang, W., Han, W., Jiang, X., Yang, S.-H. \& Parkin, S. S. P. Role of transparency of platinum-ferromagnet interfaces in determining the intrinsic magnitude of the spin Hall effect. Nat. Phys. 11, 496-503 (2015).

36. Pai, C. F., Ou, Y., Vilela-Leão, L. H., Ralph, D. C. \& Buhrman, R. A. Dependence of the efficiency of spin Hall torque on the transparency of Pt/ferromagnetic layer interfaces. 
Phys. Rev. B 92, 064426 (2015).

37. Zhang, W. et al. Spin Hall effects in metallic antiferromagnets. Phys. Rev. Lett. 113, 196602 (2014).

38. Okada, A. et al. Spin-pumping-free determination of spin-orbit torque efficiency from spin-torque ferromagnetic resonance. Phys. Rev. Appl. 12, 014040 (2019).

39. Zhu, L., Ralph, D. C. \& Buhrman, R. A. Spin-Orbit Torques in Heavy-Metal-Ferromagnet Bilayers with Varying Strengths of Interfacial Spin-Orbit Coupling. Phys. Rev. Lett. 122, 77201 (2019).

40. Feng, J. et al. Effects of oxidation of top and bottom interfaces on the electric, magnetic, and spin-orbit torque properties of Pt / Co / Al Ox trilayers. Phys. Rev. Appl. 13, 044029 (2020).

41. Pai, C. F. et al. Spin transfer torque devices utilizing the giant spin Hall effect of tungsten. Appl. Phys. Lett. 101, 122404 (2012).

42. Cho, S., Chris Baek, S., Lee, K.-D., Jo, Y. \& Park, B.-G. Large spin Hall magnetoresistance and its correlation to the spin-orbit torque in $\mathrm{W} / \mathrm{CoFeB} / \mathrm{MgO}$ structures. Sci. Rep. 5, 14668 (2015).

43. Zhu, L. et al. Strong damping-like spin-orbit torque and tunable Dzyaloshinskii-Moriya interaction generated bylLow-resistivity $\mathrm{Pd}_{1-\mathrm{x}} \mathrm{Pt}_{\mathrm{x}}$ alloys. Adv. Funct. Mater. 29, 1805822 (2019).

44. Tsai, H. et al. Electrical manipulation of a topological antiferromagnetic state. Nature 580, 608 (2020). 
436 45. Cao, J. et al. Spin-orbit torque induced magnetization switching in $\mathrm{Ta} / \mathrm{Co}_{20} \mathrm{Fe}_{60} \mathrm{~B}_{20} / \mathrm{MgO}$ structures under small in-plane magnetic fields. Appl. Phys. Lett. 108, 172404 (2016).

438 46. Baumgartner, M. et al. Spatially and time-resolved magnetization dynamics driven by spin-orbit torques. Nat. Nanotechnol. 12, 980-986 (2017).

440 441

442

443

444

445

446

447

448

449

450

451

452

453

454

455

456

457

47. Avci, C. O. et al. Unidirectional spin Hall magnetoresistance in ferromagnet/normal metal bilayers. Nat. Phys. 11, 570-575 (2015).

48. Lv, Y. et al. Large unidirectional spin Hall and Rashba-Edelstein magnetoresistance in topological insulator/magnetic insulator heterostructures. arXiv:1806.09066. (2018).

49. Guo, G. Y., Murakami, S., Chen, T.-W. \& Nagaosa, N. Intrinsic spin Hall effect in platinum: first-principles calculations. 100, 096401 (2008).

50. Sinova, J., Valenzuela, S. O., Wunderlich, J., Back, C. H. \& Jungwirth, T. Spin Hall effects. Rev. Mod. Phys. 87, 1213-1260 (2015).

\section{Acknowledgments}

This research was supported in part by ASCENT, one of six centers in JUMP, a Semiconductor Research Corporation (SRC) program sponsored by DARPA. The authors thank the NSF Center for Energy Efficient Electronics Science (E3S) and TSMC for financial support. Part of this work was performed at the Stanford Nano Shared Facilities (SNSF)/Stanford Nanofabrication Facility (SNF), supported by the National Science Foundation under award ECCS-1542152. The research at the University of Nebraska-Lincoln is supported by the National Science Foundation through the Nebraska Materials Science and Engineering Center (MRSEC, Grant No. DMR1420645). P. Q. acknowledges support from the National Research Council Research Associateship Program. S.E. and M. B. V. acknowledge funding from NSF award DMR- 
1905909, and assistance from Randy Dumas at Quantum Design with VSM measurements. The

459 authors would also like to acknowledge Dr. Carlos H. Diaz, Dr. Peng Li, and Dr. Juliet

460 Jamtgaard for fruitful discussions.

\section{Author contributions}

462 M. DC conceived, designed, and coordinated the research with contributions from M.M., S.-J.L., 463 W.T., and S.X.W. S.X.W supervised the study. M. DC grew thin films, performed XRD 464 measurement, fabricated Hall bar, ST-FMR device, carried out ST-FMR, SHH, and switching 465 measurements with contributions from Y.D., X.L., C.B., F.X., and Y-L. H. D-F. S. and E. T. 466 performed DFT calculations. V.D.H., A. H., and W. W. carried out TEM and EDS studies. A.V. 467 performed pole figure measurements. M. B.V. and S.E. performed magnetometry measurements. 468 P.Q., B.K., and J.B. performed PNR measurements and modelling. M. DC performed LLG 469 simulations. M. DC performed data analysis and wrote the manuscript with contributions from 470 D-F. S, P.Q., A.V., and S.X.W. All authors discussed the results and commented on the 471 manuscript.

\section{Online content}

473 Any methods, additional references, Nature Research reporting summaries, source data,

474 Supplementary, supplementary information, acknowledgements, peer review information; details 475 of author contributions and competing interests; and statements of data and code availability are 476 available online.

\section{Competing Interests}

478 The authors declare no competing interests.

\section{Data Availability}

480 The data that support the findings of this study are available from the corresponding authors on 481 reasonable request. 


\section{${ }_{483}$ Methods}

484

485

486

487

488

489

490

491

492

493

494

495

496

497

498

499

500

501

502

503

504

\section{Sample growth and thin film characterization}

Manganese Palladium $\left(\mathrm{MnPd}_{3}\right)$ thin films for the magneto-transport, SOT characterization, and magnetization switching were grown on $\mathrm{Si} / \mathrm{SiO}_{2}$ substrate at room temperature by sputtering a composite $\operatorname{MnPd}_{2}\left(99.99 \%\right.$ pure) target in AJA sputtering system ${ }^{51}$ with a base pressure of $2.0 \times 10^{-8}$ Torr. $\mathrm{MnPd}_{3}, \mathrm{CoFeB}, \mathrm{Pt}$, and Ta were dc sputtered at $25 \mathrm{~W}$ power with deposition rates $0.27,0.056,0.27$, and $0.07 \AA / \mathrm{s}$, respectively. The $\mathrm{MgO}$ layer was $\mathrm{rf}$ sputtered at $50 \mathrm{~W}$ power with a deposition rate of $0.013 \AA / \mathrm{s}$.

The thin section for transmission electron microscopy (TEM) was prepared by the focus ion beam with FEI Helios Nanolab $660^{51}$. The TEM analysis was carried out by a Hitachi HF5000 equipped with a probe aberration corrector, under $200 \mathrm{kV}$. Bright field (BF), secondary electron (SE) and Annular (both medium and high angle) dark field (ADF) images were captured simultaneously in scanning TEM (STEM) mode. High resolution energy dispersive X-ray spectroscopy (EDS) mapping and line scan were performed by an Oxford Instruments large solid angle $(2.0 \mathrm{sr})$ dual windowless detectors ${ }^{51}$.

\section{Device fabrication and electrical characterization}

The multilayer thin films were patterned into Hall bars for the quantum transport, SHH, and magnetization switching experiments using an optical lithography process. The width and length of Hall bar was $10 \mu \mathrm{m}$ and length $130 \mu \mathrm{m}$, respectively. The separation between the Hallcrosses in Hall bar device is $37 \mu \mathrm{m}$. For the ST-FMR measurement thin films were patterned into rectangular strips of dimension 25-30 $\mu \mathrm{m}$ length and width, respectively. After the first step of photolithography, the Ar ion mill was used to define the device geometry. Ti $(10 \mathrm{~nm}) / \mathrm{Au}(150$ 
$505 \mathrm{~nm}$ ) thick electrical contacts were deposited after the second step of lithography using KJL e506 beam evaporator. A physical property measurement system (PPMS) ${ }^{51}$ was used to measure the 507 electrical properties. Two Lockins SR830 ${ }^{51}$ and 6221 current source $^{51}$ were used to probe first 508 and second harmonic voltage and supply current, respectively. An Agilent HP $83620 \mathrm{~B}^{51}$ was 509 used to supply the r.f. current for the ST-FMR measurements. The d.c. voltage was measured 510 with a Keithley 2000 multi-meter ${ }^{51}$. 

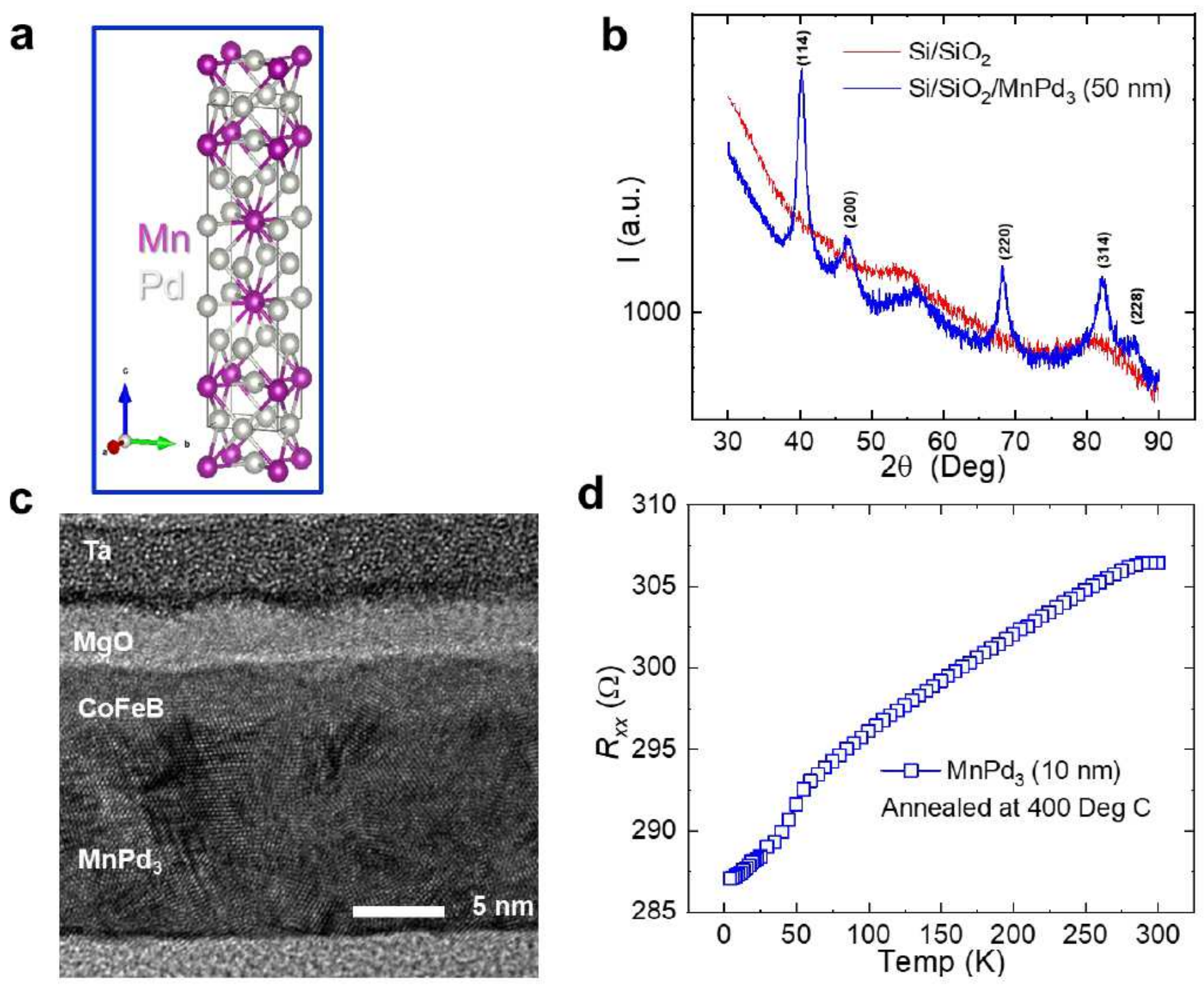

Figure 1

Characterization of MnPd3 thin film: a, Schematic diagram of D023 MnPd3 unit cell. b, XRD of $\mathrm{Si} / \mathrm{SiO} 2 / \mathrm{MnPd} 3(50 \mathrm{~nm})$ film, post-annealed at $400^{\circ} \mathrm{C}$ for $30 \mathrm{~min}$. c, Cross-section TEM image of $\mathrm{Si} / \mathrm{SiO} 2 / \mathrm{MnPd} 3(10 \mathrm{~nm}) / \mathrm{CoFeB}(5 \mathrm{~nm}) / \mathrm{MgO}(2 \mathrm{~nm}) / \mathrm{Ta}(2 \mathrm{~nm})$ sample. d, Four terminal resistance as a function of temperature of $\mathrm{Si} / \mathrm{SiO} 2 / \mathrm{MnPd} 3(10 \mathrm{~nm})$ sample. 

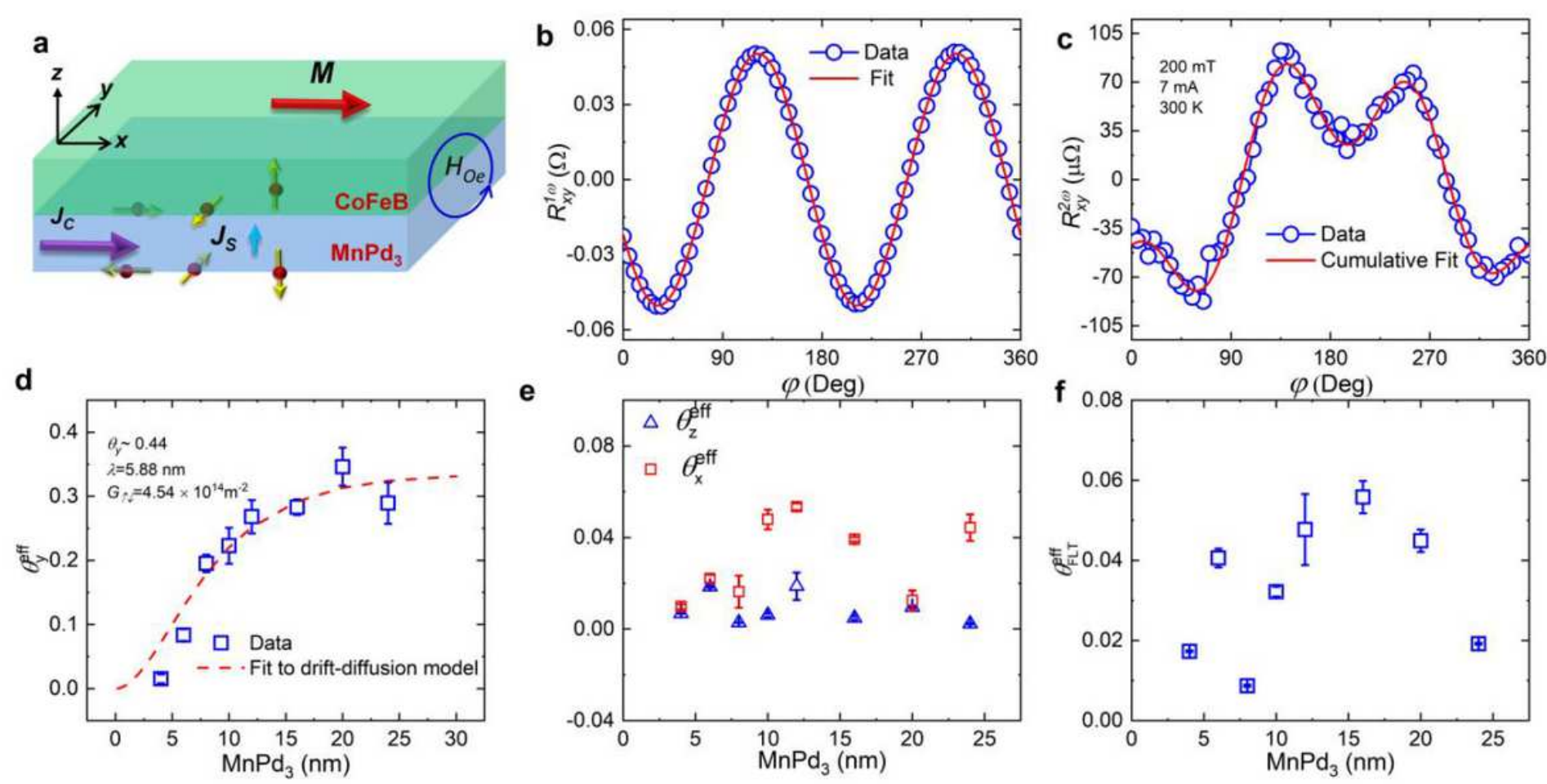

Figure 2

SOT characterization using Second Harmonic Hall (SHH) technique on Si/SiO2/MnPd3 (x nm)/CoFeB (5 $\mathrm{nm}) / \mathrm{MgO}(2 \mathrm{~nm}) / \mathrm{Ta}(2 \mathrm{~nm})$ : a, Schematic diagram showing in-plane charge current generated spin current with spin polarizations along three axes. The red spheres represent electrons and yellow arrows

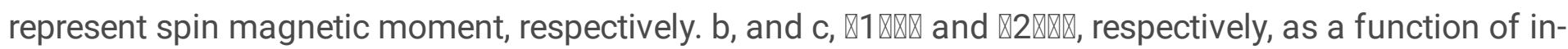
plane magnetic field rotation at a fixed amplitude of $200 \mathrm{mT}$ in MP12 sample. $d, e$, and f, Effective spin torque efficiency due to the in-plane anti-damping-like, in-plane and out-of-plane anti-damping-like and field-like torques, respectively, as a function of MnPd3 film thickness. The Hall bar device used for this measurement was $10 \mu \mathrm{m}$ wide and $130 \mu \mathrm{m}$ long, respectively. 

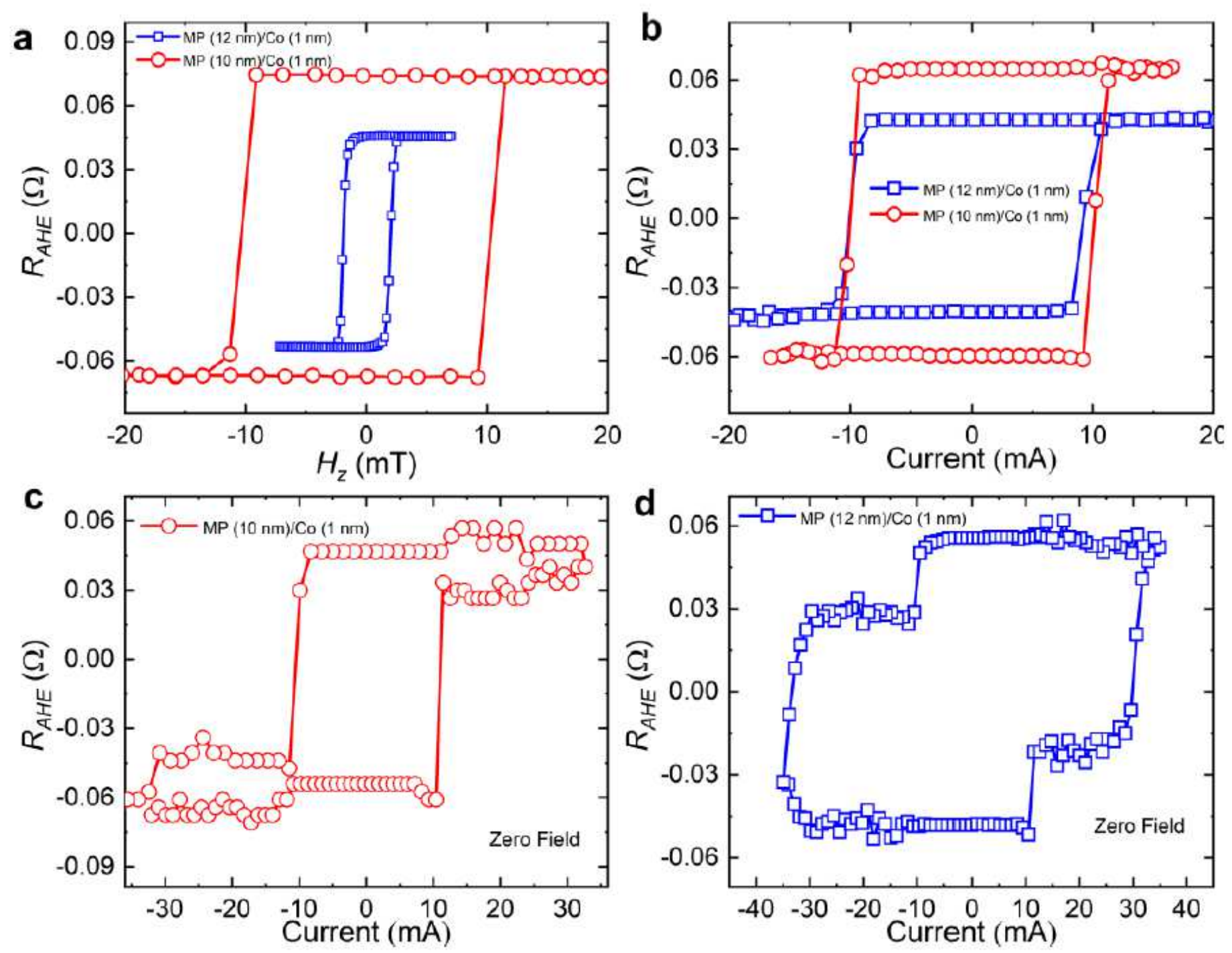

Figure 3

Demonstration of external magnetic field-free out-of-plane magnetization switching via z-spin polarization generated anti-damping spin-orbit torque: $a$, The anomalous Hall resistance as a function of out-of-plane external field. b, Switching of perpendicular Co layer via SOT under the application of -8 mT and -20 mT along x-direction for MP $(10 \mathrm{~nm}) / \mathrm{Co}(1 \mathrm{~nm})$ and MP $(12 \mathrm{~nm}) / \mathrm{Co}(1 \mathrm{~nm})$ samples, respectively. $c$, and d, Field-free perpendicular Co layer switching via out-of-plane anti-damping-like torque generated by z-spin polarization. The Hall bar with a length of $130 \mu \mathrm{m}$ and a width of $10 \mu \mathrm{m}$ was used for the magnetization switching experiment. 

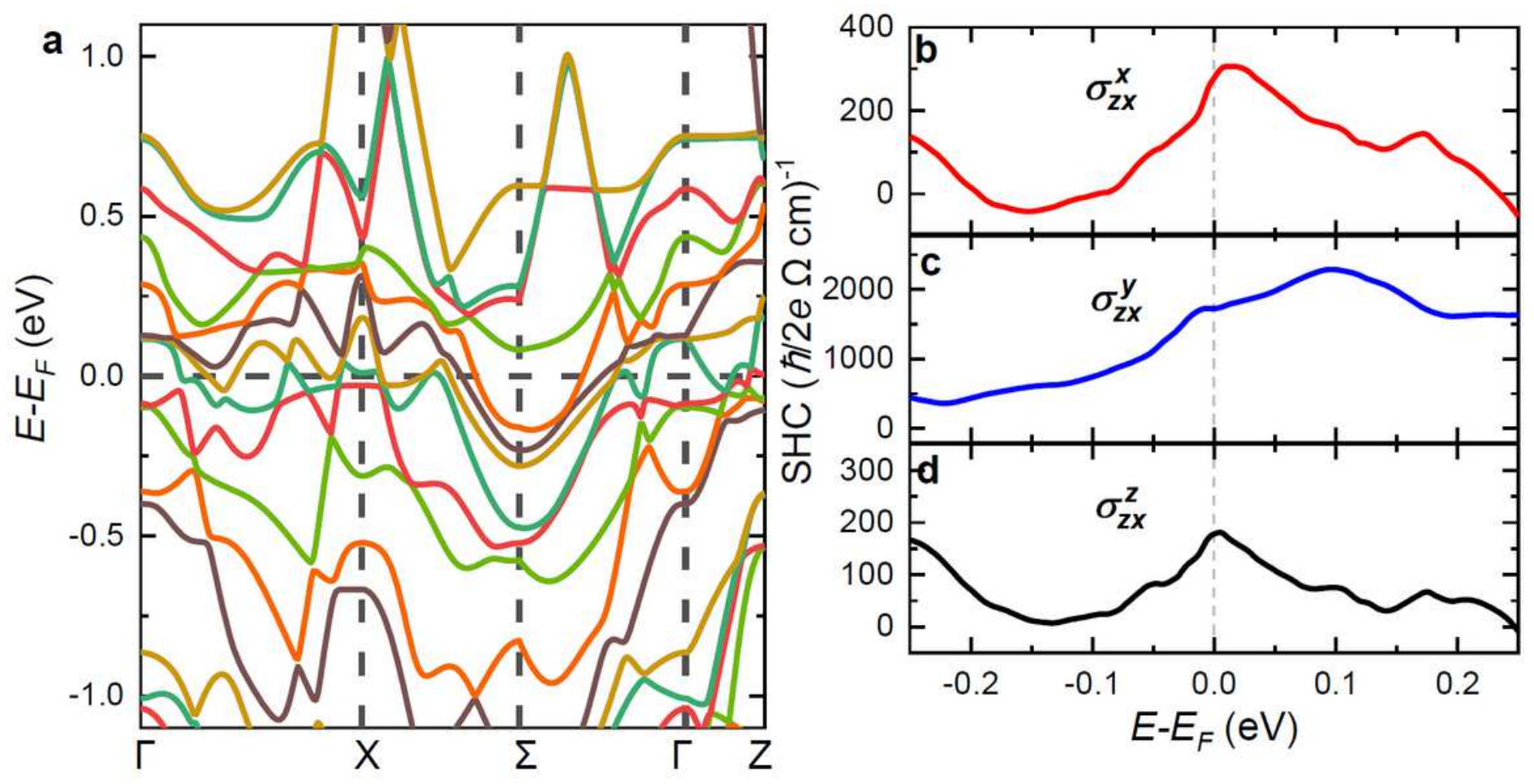

Figure 4

Effect of (114) texture on the spin polarization. a, The calculated band structure of stoichiometric MnPd3

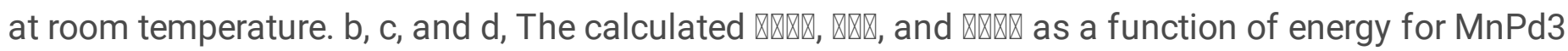
(114) film, where the $x$ axis is oriented along the [01] direction.

\section{Supplementary Files}

This is a list of supplementary files associated with this preprint. Click to download.

- SupplementarylnformationV7.pdf 